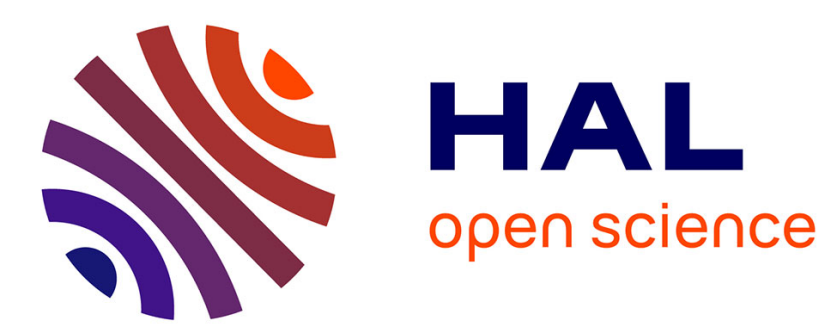

\title{
Discrete Lot Sizing and Scheduling using Product Decomposition into Attributes
}

Céline Gicquel, Nicolas Miègeville, Michel Minoux, Yves Dallery

\section{To cite this version:}

Céline Gicquel, Nicolas Miègeville, Michel Minoux, Yves Dallery. Discrete Lot Sizing and Scheduling using Product Decomposition into Attributes. Computers and Operations Research, 2009, 36 (9), pp.2690-2698. 10.1016/j.cor.2008.11.017 . hal-01170200

\section{HAL Id: hal-01170200 \\ https://hal.science/hal-01170200}

Submitted on 3 Feb 2016

HAL is a multi-disciplinary open access archive for the deposit and dissemination of scientific research documents, whether they are published or not. The documents may come from teaching and research institutions in France or abroad, or from public or private research centers.
L'archive ouverte pluridisciplinaire HAL, est destinée au dépôt et à la diffusion de documents scientifiques de niveau recherche, publiés ou non, émanant des établissements d'enseignement et de recherche français ou étrangers, des laboratoires publics ou privés. 


\title{
Discrete lot sizing and scheduling using product decomposition into attributes
}

\author{
C. Gicquel ${ }^{a, *}$, N. Miègeville ${ }^{b}$, M. Minoux $^{c}$, Y. Dallery ${ }^{a}$ \\ ${ }^{a}$ Ecole Centrale Paris, Laboratoire Genie Industriel, Grande Voie des Vignes \\ 92290 Chatenay-Malabry, France \\ ${ }^{b}$ Saint-Gobain Recherche, 39 quai Lucien-Lefranc, 93303 Aubervilliers, France \\ ${ }^{c}$ Laboratoire d'Informatique de Paris 6, 4 place Jussieu, 75005 Paris, France
}

\begin{abstract}
A production planning problem, known as the Discrete Lot sizing and Scheduling Problem with sequence-dependent changeover costs, is considered. We propose a new way of modelling the production system based on the use of a multi attribute product structure encountered in many industrial situations. The basic idea is to describe the products as combinations of physical attributes and to exploit this description to reduce the size of the mixed-integer program to be solved. The results of our computational experiments show the practical usefulness of the proposed formulation which leads to significantly improved efficiency in the solution process.
\end{abstract}

Keywords: Production Planning, Lot Sizing, Mixed Integer Programming

\footnotetext{
${ }^{*}$ Corresponding author.
}

Tel.: +331411313 88; fax: +33141131272.

E-mail address: celine.gicquel@ecp.fr 


\section{Introduction}

A wide variety of models for production planning and inventory management has been investigated. Among them, capacitated lot sizing models aim at determining a minimal cost production schedule complying with given capacity restrictions and such that demand for all products is satisfied without backlogging. Lot sizing problems can be broadly classified by the number of products, capacitated resources and product levels involved. The present work pertains to multi-item single-resource single-level lot-sizing models: multiple items assumed to be independent to one another (i.e. not linked by parentcomponent relationships) are to be produced on a single constrained resource. Moreover all problem parameters are supposed to be deterministically known.

A further important distinction among deterministic lot-sizing models can be made based on considerations on the demand (see e.g. [16]):

- On one hand, there are models such as the Economic Lot Scheduling Problem (ELSP) where the demand rate is supposed to be constant and a continuous time scale and infinite time horizon are used. Early contributions on the ELSP can be found for instance in [2] and [7].

- On the other hand, some models assume a dynamic, time-varying demand and use a discrete time scale and finite time horizon. In such models, time-dependent demand is specified on a period-by-period basis. Recent overviews on the literature about this line of research can be found in [6], [12], [13] and [32] .

In the present paper, we focus on a capacitated dynamic lot-sizing problem known as the Discrete Lot sizing and Scheduling Problem (DLSP). The DLSP is based on several key assumptions (see e.g. [8]):

- Demand for products is deterministically known and time-varying.

- The production plan is established for a finite time horizon subdivided into several discrete periods.

- At most one item can be produced per period ("small bucket" model) and the facility processes either one product at full capacity or is completely idle ("all-ornothing" assumption).

- Costs to be minimized are the inventory holding costs and the changeover costs.

In lot sizing problems, the changeover costs to be incurred when the production of a new lot begins can depend either on the next item only (sequence-independent case) or on both the previous and the next items (sequence-dependent case). We consider 
here the (more difficult) case of sequence-dependent changeover costs. In this paper, we assume zero changeover times. However the integration of positive changeover times is an important extension of our model and is currently being worked on.

The reader is referred to [33] for a recent literature review on scheduling and lot sizing with sequence-dependent setup. In the sequel, we discuss more specifically contributions on the DLSP with sequence-dependent changeover costs. In [9] and [24], the problem is reformulated as a Travelling Salesman Problem with Time Windows and is solved using either Lagrangian relaxation or a dynamic programming-based algorithm. The authors of [14] show the equivalence between the DLSP with sequence-dependent changeover costs and the Batch Sequencing Problem (BSP) and use a specific branch-and-bound type algorithm for solving the BSP to optimality. In these papers, the number of items considered in the computational experiments is relatively small (no more than 10 items) whereas the horizon length can be up to 100 periods. In a recent paper, [32] proposes to strengthen a basic MIP (mixed-integer programming) formulation of the DLSP with sequence-dependent changeover costs using both a reformulation of the changeover variables and valid inequalities. Thanks to this strengthened formulation, the lower bounds provided by the linear relaxation of the problem are significantly better, enabling a Branch \& Bound type procedure to solve the problem more efficiently. However, as pointed out by [1], the large number of variables needed in the reformulation to handle changeovers is an important drawback of this approach.

The purpose of the present paper is to propose a new way of modelling the production system to be planned by properly exploiting a multi attribute product structure frequently encountered in industrial applications. The basic idea of the new model originates from the observation that products can usually be described in terms of a set of physical attributes such as color, dimension, quality level... If this is possible, each item to be produced will be identified, not only by a unique index as it is usually done, but also by a $M$-tuple, each component of which indicates the value of the corresponding attribute for the given item. When such a multi attribute product structure can be exhibited in the industrial context under study, we propose to exploit it to reduce the size of the mixed integer program to be solved. This is achieved by looking at changeovers at an aggregate level using the relevant physical attributes instead of considering each individual changeover between items. By doing so, we are able to significantly reduce the number of changeover variables and associated constraints in the MIP formulation, while maintaining the quality of the bounds provided by the linear relaxation of the problem. We extend the approach used by [32] to derive valid inequalities for the resulting mixedinteger linear program. Computational results show that exact optimal solutions can in general be obtained more efficiently with the new model as compared with previously 
described approaches.

The paper is organized as follows. In section 2, we introduce the strengthened reformulation proposed by [32] for the DLSP with sequence-dependent changeover costs. In our computational experiments, we use it as a reference for comparison with our model. In section 3, we describe our proposal to model the production system using product attributes and discuss our main assumptions. When these assumptions hold, we propose in section 4 to exploit them to formulate the DLSP with sequence-dependent changeover costs with a reduced number of variables and constraints. Results of computational experiments carried out on a large number of randomly generated instances to validate our approach are reported in section 5. Section 6 presents the concluding remarks and discussions for future investigations.

\section{A strong formulation for the DLSP with sequence- dependent changeover costs}

In this section, we recall a strong formulation for the DLSP with sequence-dependent changeover costs. This formulation was presented by [15] for the variant of the DLSP

referred to as CSLP (Continuous Setup Lot sizing Problem), where the all-or-nothing assumption is relaxed. More recently, [1] and [32] propose to use it to solve the DLSP with sequence-dependent changeover costs. We next discuss a further strengthening of this formulation obtained by exploiting valid inequalities developed by [30].

We wish to optimize the production schedule for a set of $N$ items over an horizon featuring $T$ planning periods. A period is indexed by $t=1, \ldots, T$, an item by $k=0, . ., N$. We agree to use item $k=0$ to represent idle periods.

We use the following notation for the parameters:

- $D_{k t}$ : demand (in units) for item $k$ in period $t$,

- $P_{k t}$ : production capacity (in units per period) for item $k$ in period $t$,

- $h_{k}$ : holding costs per unit and period for item $k$,

- $c_{k l}$ : changeover costs from item $k$ to item $l$.

Decision variables are defined as follows:

- $I_{k t}$ : inventory level corresponding to item $k$ at the end of period $t$,

- $y_{k t}$ : setup variables. $y_{k t}=1$ if the resource is setup for item $k$ in period $t$, and 0 otherwise, 
- $w_{k l t}$ : changeover variables. $w_{k l t}=1$ if the resource is switched from item $k$ to item $l$ at the beginning of period $t$, and 0 otherwise.

With this notation, [1] propose to formulate the DLSP with sequence-dependent changeover costs as follows:

(DLSP1)

$$
\begin{aligned}
& \min \sum_{k=1}^{N} \sum_{t=1}^{T} h_{k} I_{k t}+\sum_{k=0}^{N} \sum_{l=0}^{N} \sum_{t=1}^{T} c_{k l} w_{k l t} \\
& \forall k, \forall t, I_{k t}=I_{k, t-1}+P_{k t} y_{k t}-D_{k t} \\
& \sum_{k=0}^{N} y_{k 0}=1 \\
& \forall k, \forall t, y_{k, t-1}=\sum_{l=0}^{N} w_{k l t} \\
& \forall l, \forall t, y_{l t}=\sum_{k=0}^{N} w_{k l t} \\
& \forall k, \forall l, \forall t, w_{k l t} \geq 0 \\
& \forall k, \forall t, I_{k t} \geq 0 \\
& \forall k, \forall t, y_{k t} \in\{0,1\}
\end{aligned}
$$

The objective, minimizing the sum of inventory holding costs and changeover costs, is expressed by (1). Changeover costs $c_{k l}$ are incurred between two successive production batches of item $k$ and item $l$, in the first period of production of item $l$. Constraints (2) express the inventory balance. The "all-or-nothing" assumption is enforced by the term $P_{k t} y_{k t}$ in the equality: if the resource is setup for $k$ in period $t$, then all the available capacity is used and the production quantity of item $k$ must be equal to $P_{k t}$. (3) is also linked to the "all-or-nothing" assumption: together with constraints (4)-(5), they ensure that in each period, the resource either produces a single product at full capacity, or is idle (i.e $y_{0 t}=1$ ). Equalities (4) and (5) link the setup variables with the changeover variables. (4) guarantee that item $k$ can be produced in period $t-1$ if and only if a changeover from $k$ to another item $l$ (possibly $l=k$ ) takes place at the beginning of period $t$. Similarly, (5) guarantee that item $l$ can be produced in period $t$ if and only if a changeover from another item $k$ (possibly $k=l$ ) to item $l$ takes place at the beginning of period $t$. (6) state the non-negativity of the changeover variables: observe, as pointed out by [1], that thanks to constraints (3)-(5) and (8), there is no need to define variables $w_{k l t}$ as binary variables. The set of constraints (2) and (7) ensure that demand for each item is fulfilled without backlogging. The binary character of the setup variables is represented 
by (8).

As suggested by [32], the formulation DLSP1 can be further strengthened using a family of strong valid inequalities developed by [30] for the single-item DLSP with WagnerWhitin costs, constant capacity and no backlogging. Under these assumptions, demand can be measured in terms of how many units can be produced during one production period, i.e. the production capacity and demand quantity can be normalized to one unit per period without loss of generality: $d_{k t} \in\{0,1\}$ and $P_{k t}=1$

In order to present the valid inequalities developed by [30], we first introduce some additional notation:

- $\tilde{D}_{k, t, \tau}$ : cumulated demand for item $k$ in the interval $\{t, \ldots, \tau\}$. Thanks to the normalization, demand on item $k$ is binary so that $\tilde{D}_{k, t, \tau}$ is equal to the number of positive demand periods for $k$ in $\{t, \ldots, \tau\}$.

- $S_{k, q}: q^{t h}$ positive demand period for item $k$. Note that $S_{k, \tilde{D}_{k, 1, t}+q}$ denotes the $q^{\text {th }}$ period with positive demand after period $t$.

We also introduce the start-up variables $z_{k t}$ defined as:

$$
z_{k t}=\left\{\begin{aligned}
1 & \text { if the production of a new lot of item } k \text { begins at period } t, \text { i.e. if } \\
& \text { a start-up for item } k \text { takes place at the beginning of period } t \\
0 \text { otherwise. } &
\end{aligned}\right.
$$

These variables are linked to the changeover variables by the equations:

$$
\forall k, \forall t, z_{k t}=\sum_{l: l \neq k} w_{l k t}
$$

With this notation, the following inequations (10) are valid inequalities for the DLSP with sequence-dependent changeover costs:

$$
\forall t, \forall k, \forall p \in\left\{0 \ldots \tilde{D}_{k, t+1, T}\right\}, I_{k t} \geq \sum_{q=1}^{p}\left(1-y_{k, t+q}-\sum_{\tau=t+q+1}^{S_{k, \tilde{D}_{k, 1, t}+q}} z_{k \tau}\right)
$$

We briefly explain the underlying idea. First note that $y_{k, t+q}+\sum_{\tau=t+q+1}^{S_{k, \tilde{D}_{k, 1, t}+q}} z_{k \tau}=0$ if and only if the resource is not setup for item $k$ in period $t+q$ and no startup for $k$ takes place between the period $t+q+1$ and the period where the $q^{t h}$ demand after period $t$ occurs, i.e. if and only if no production of item $k$ is possible in the interval $\left\{t+q, \ldots, S_{k, \tilde{D}_{k, 1, t}+q}\right\}$. In this case, the quantity needed to satisfy the $q^{\text {th }}$ demand after period $t$ should be in stock at the end of period $t$. Thus we see that constraints (10) force an increase of the stock 
of item $k$ at the end of period $t$ by one for each index $q$ for which no production occurs in the interval $\left\{t+q, \ldots, S_{k, \tilde{D}_{k, 1, t}+q}\right\}$. The reader is referred to [30] for a detailed proof of the validity of (10). In our computational experiments, we add these valid inequalities to the formulation according to a cutting-plane generation strategy (for details see section 4.2). The resulting strengthened formulation is denoted DLSP1*.

As pointed out by [1], an important drawback of the DLSP1 formulation is that the number of variables needed in the formulation to handle changeovers, $(N+1)^{2} T$, grows very rapidly with problem size. In the sequel, we present one way to avoid this issue in certain situations, namely when products can be described as combinations of a number of physical attributes.

\section{The DLSP with products described as combina- tions of physical attributes}

In most papers dealing with lot sizing problems, each individual item to be produced is described with a single index ( $k$ in the formulation presented above) and is considered independently of the other items. However, in many industrial situations, the items to be produced differ in their physical characteristics or attributes (e.g. color, dimensions, shape, mixture composition, quality level...). As explained by [21] for the case of a metals plant, such physical attributes often contribute significantly to the sequence dependency of changeover costs. Moreover it is frequently the case that many items share a common value for some attribute so that we can define a (small) finite number of possible values for each attribute. In the sequel, we propose to describe each item to be produced as well as the resource setup state using the physical attributes of the products and to look at changeovers at an aggregate level using the relevant physical attributes instead of considering each individual changeover between items. By doing so, we are able to simplify the production planning process with respect to:

- the evaluation of changeover costs. Changeovers costs are most often estimated by human production experts. In the presence of a multi attribute product structure, it may be easier for them to evaluate changeover costs attribute by attribute rather than item by item. This was experienced by the authors in the industrial application described in [19].

- the resolution of the optimization problem. Thanks to the use of a multi-attribute product structure, we are able to reduce the size of the obtained mixed integer program and thus to make it easier to solve with a standard MIP software. 
The idea of exploiting a multi attribute product structure may be used in a variety of modelling environments. We focus here on its application on a particular type of lot sizing problems, namely the DLSP, but the present work could be extended to deal with a closely related dynamic lot sizing problem known as the General Lot sizing and Scheduling Problem or GLSP (see e.g. [17]).

In section 4, we derive a new formulation for the DLSP which is likely to be solved more efficiently using standard MIP software. In order to do this, we need to make several assumptions on the production system.

\subsection{Main assumptions}

1. We suppose that each item to be produced can be described by a set of physical attributes, each of them takes a finite number of discrete values. We also suppose that each item is uniquely identified thanks to a $M$-tuple, each component of which gives the value of the corresponding attribute for the given item.

2. We assume that the setup state of the resource can also be described using product attributes. Thus, we will not describe the setup state of the resource by indicating the item that the resource is able to produce, but by indicating, for each attribute, for which value of this attribute the resource is setup. The resource setup state will therefore also be described by a $M$-tuple, each component of which gives the value of the corresponding attribute for the present state of the resource. To ensure consistency, it should be understood that a given item can be produced on the resource if and only if the resource is setup with the correct value for every attribute.

3. We assume that we are able to evaluate the changeover costs on the resource for each attribute separately. This means that given an attribute and two possible values for this attribute, we are able to evaluate the cost of a changeover from one value to the other and that this cost does not depend on the setup state of the resource with respect to the other attributes.

4. We need to specify how the costs relative to different attributes will combine, i.e. how we will compute changeover costs when changeovers for different attributes happen simultaneously on the resource. In this paper, we consider the case where the global changeover costs is the sum of all individual changeover costs for the different attributes. Another possible assumption is that global changeover costs equal the maximum of the individual costs.

Thanks to these assumptions, we will be able to decide about the production plan on the resource using the product attributes. In this case, the production plan will consist 
of a set of parallel sequences, one for each attribute. Each of these sequences indicates, for every planning period, for which value of the corresponding attribute the resource is setup. Thus, in each planning period, combining the values for the different attributes, we will be able to deduce the item for which the resource is setup. A detailed mathematical programming formulation is proposed in section 4 , but in order to illustrate the usefulness of the new model, we first discuss some industrial situations where it appears to be well suited.

\subsection{Possible industrial applications}

In order to illustrate the practical relevance of the proposed model, we provide examples of industrial situations found in the literature where using physical attributes to describe the products is appropriate.

- In [4] and [27], a production planning problem for a bottle filling line is considered. For this type of production line, two physical attributes of the products have to be taken into account: the size or shape of the bottle and the liquid used to fill it. Hence each item can be described by means of two attributes: the bottle size/shape and the liquid to be used. Each individual item would be described by a pair $\left(i_{1}, i_{2}\right)$ where $i_{1}$ is the index of the corresponding bottle size/shape and $i_{2}$ the index of the corresponding liquid.

- [5] discuss a lot sizing problem they found in an automated foundry. Each item to be produced can be described by two attributes: the type of metal alloy it is made of and the shape it takes from the used mould. Here we could use as well a pair $\left(i_{1}, i_{2}\right)$ where $i_{1}$ would give the index of the alloy type and $i_{2}$ the index of the mould shape.

- [26] study a production planning problem arising in the textile industry in a company producing acrylic fibers. The authors report that two physical characteristics of the products have an impact on the scheduling of the plant spinning unit, namely the fiber composition and their diameter. Thus, we could use a pair $\left(i_{1}, i_{2}\right)$ to describe each item: $i_{1}$ would refer to the fiber composition and $i_{2}$ to its diameter.

- [19] considers the production planning problem for a float glass production line. Here each item (a glass sheet) can be described using several physical characteristics: glass color and quality, dimensions of the sheet (thickness, width and length). An item could thus be described using a 5-tuple, with components corresponding to the color, quality and dimensions of the corresponding glass sheet. In fact, the pro- 
duction system model studied in the present paper was suggested in this particular context of application.

Though far from being exhaustive, the above list is a good indication of the wide applicability of the model proposed here. Moreover the proposed model could also prove useful for the frequently encountered situation where changeover costs have a majorminor setup structure. This type of structure is mainly found when the items to be produced can be grouped into families. In this case, there is a large changeover involved with setting up the resource to produce a given family of items (major setup) and an additional smaller changeover is required between two items belonging to the same family (minor setup). This situation is described for instance in [10], [11], or [23] for the case of a time-varying demand (i.e. for dynamic lot sizing problems such as the DLSP) as well as in [4], [20], [21] or [31] for the case of a constant demand (i.e. for the ELSP). Such a major-minor setup structure could be modelled using a multi attribute product structure. Namely, each item could be described using two attributes: the first one corresponding to the product family and the second one being used to identify the individual item within the family.

Our model is also capable of handling the situations where there is a change of magnitude within the values of a given physical attribute $m$, i.e. when the indices $i \in\left[0, V^{m}\right]$ correspond to values of $m$ belonging to different orders of magnitude. Namely, in this case, the corresponding changeover costs are likely to be sequence-dependent: there will be a large changeover between two values of $m$ belonging to different orders of magnitude and a smaller changeover between two values belonging to the same order of magnitude. In our model, we allow sequence-dependent changeover costs between values of a given physical attribute and are thus able to represent such situations without making any additional assumptions.

\section{A formulation for the DLSP with product at- tributes and sequence-dependent changeover costs}

\subsection{Basic formulation}

We now present a formulation for the DLSP with product attributes and sequencedependent changeover costs. This formulation can be used to solve the DLSP when a product description using physical attributes is possible and when the assumptions discussed in section 3.1 hold.

We use the same notation as in section 2 for the parameters relative to items: 
- $D_{k t}$ : demand for item $k$ in $t$,

- $P_{k t}$ : production capacity for item $k$ in $t$,

- $h_{k}$ : holding costs per unit and period for $k$.

We assume that each item can be described using $M$ physical characteristics or attributes. Correspondence between products and attributes is given by a matrix $\mathcal{A}$ of dimensions $M \times(N+1) . \mathcal{A}_{m k}$ represents the value of the attribute $m$ for product $k$ and the $k^{t h}$ column of $\mathcal{A}$ gives the $M$-tuple describing product $k$ in terms of product attributes. For each attribute $m$, we have:

- a set of possible values: $i \in\left[0, V^{m}\right]$

- a changeover cost matrix: $\mathcal{C}^{m}$. $C_{i j}^{m}$ is the cost of a transition from the value $i \in$ $\left[0, V^{m}\right]$ to the value $j \in\left[0, V^{m}\right]$ of attribute $m$.

We agree to use the $M$-tuple $(0,0, \ldots, 0)$ to describe the item $k=0$ : i.e $\forall m, \mathcal{A}_{m 0}=0$.

We use the following decision variables:

- $I_{k t}$ : inventory level corresponding to item $k$ at the end of period $t$,

- $y_{k t}$ : setup variables at the item level. $y_{k t}=1$ if the resource is setup for item $k$ in period $t$, and 0 otherwise,

- $w_{i j t}^{m}$ : changeover variables at the attribute level. $w_{i j t}^{m}=1$ if a switch from the value $i$ to the value $j$ of attribute $m$ takes place at the beginning of period $t$, and 0 otherwise.

Under the assumption that changeover costs related to different attributes are added whenever two transitions occur simultaneously (see assumption 4 in section 3.1), the DLSP can be formulated as follows:

(DLSP2)

$$
\begin{aligned}
& \min \sum_{k=1}^{N} \sum_{t=1}^{T} h_{k} I_{k t}+\sum_{m=1}^{M} \sum_{i=0}^{V^{m}} \sum_{j=0}^{V^{m}} \sum_{t=1}^{T} C_{i j}^{m} w_{i j t}^{m} \\
& \forall k, \forall t, I_{k t}=I_{k, t-1}+P_{k t} y_{k t}-D_{k t} \\
& \sum_{k=0}^{N} y_{k 0}=1 \\
& \forall m, \forall i \in\left[0, V^{m}\right], \forall t, \sum_{k \operatorname{st} \mathcal{A}_{m k}=i} y_{k, t-1}=\sum_{j=0}^{V^{m}} w_{i j t}^{m}
\end{aligned}
$$




$$
\begin{aligned}
& \forall m, \forall j \in\left[0, V^{m}\right], \forall t, \sum_{k \text { st } \mathcal{A}_{m k}=j} y_{k t}=\sum_{i=0}^{V^{m}} w_{i j t}^{m} \\
& \forall m, \forall(i, j) \in\left[0, V^{m}\right] \times\left[0, V^{m}\right], \forall t, w_{i j t}^{m} \geq 0 \\
& \forall k, \forall t, I_{k t} \geq 0 \\
& \forall k, \forall t, y_{k t} \in\{0,1\}
\end{aligned}
$$

The objective, minimizing the sum of changeover costs and inventory holding costs, is expressed by (11). Note that inventory holding costs are computed item by item whereas changeover costs are computed attribute by attribute. Constraints (12) express the inventory balance. Combined with the non negativity constraints (17), they prevent any backlogging. (13), together with constraints (14)-(15), guarantee that in each period the resource either produces a single item or is idle.

Equalities (14) and (15) link the setup variables with the changeover variables. First note that the term $\sum_{k \text { st } \mathcal{A}_{m k}=i} y_{k t}$ equals 1 if and only if an item $k$ requiring the resource to be setup for the value $i$ of the attribute $m$ is produced in period $t$, i.e. if and only if the resource is setup for the value $i$ of attribute $m$ in period $t$. Thus (14) guarantee that the resource is setup for the value $i$ of attribute $m$ in period $t-1$ if and only if a changeover from value $i$ to another possible value $j$ of attribute $m$ (possibly $j=i$ ) takes place at the beginning of period $t$. Similarly, (15) guarantee that the resource is setup for the value $j$ of attribute $m$ in period $t$ if and only if a changeover from another possible value $i$ of attribute $m$ (possibly $i=j$ ) to value $j$ takes place at the beginning of period $t$. The non negativity of the changeover variables is stated by (16) and the binary character of the setup variables is expressed by (18).

The formulation DLSP2 can be easily modified to consider the other possible assumption about the combination of costs relative to different attributes, i.e. the assumption that global changeover costs equal the maximum of the individual costs. This can be done by defining additional continuous variables $C_{t}$ to evaluate the changeover costs to be incurred at the beginning of each period $t$. In this case, the DLSP can be formulated as follows:

(DLSP2 MAX)

$$
\begin{aligned}
& \min \sum_{k=1}^{N} \sum_{t=1}^{T} h_{k} I_{k t}+\sum_{t=1}^{T} C_{t} \\
& \text { s.t. } \forall m, \forall t, C_{t} \geq \sum_{i=0}^{V^{m}} \sum_{j=0}^{V^{m}} C_{i j}^{m} w_{i j t}^{m} \\
& \text { and }(12)-(18)
\end{aligned}
$$


In the sequel, we assume that global changeover costs equal the sum of the individual costs and thus use the formulation DLSP2. However similar results could be obtained with the other assumption.

Let us now compare the number of changeover variables in the formulations DLSP1 and DLSP2. For the sake of simplicity, we do not take into account the item $k=0$ in the comparison. As shown in section 2, in this case, DLSP1 includes $N^{2} T$ changeover variables, one for each possible pair of items and for each period. Note that when the product description using attributes is possible, we can compute the number of products as the number of possible combinations obtained by choosing for each attribute $m$ one value out of $V^{m}$. Thus we have: $N^{2}=\left(\prod_{m=1}^{M} V^{m}\right)^{2}$. Now, as can be seen above, in the DLSP2 formulation, there are $\sum_{m=1}^{M}\left(V^{m 2}\right) T$ changeover variables, one for each pair of possible values of each attribute and for each period. In most cases where the product description using attributes will be implemented, we will have: $\sum_{m=1}^{M}\left(V^{m 2}\right) \ll$ $\left(\prod_{m=1}^{M} V^{m}\right)^{2}$, thus leading to a significant reduction in the number of changeover variables needed in the formulation.

In words, in the proposed model, we do not consider each individual changeover between items, but rather look at changeovers at a more aggregate level using product attributes. By doing so, we are able to significantly reduce the size of the mixed integer linear programm to be solved (e.g. using a Branch \& Bound procedure).

\subsection{Strengthening the formulation with valid inequalities}

As for the DLSP1 formulation, the DLSP2 formulation can be further strengthened under the assumption of Wagner-Whitin costs, constant capacity and no backlogging. This can be achieved by extending the inequalities (10) to the DLSP2 formulation. In order to do this, we define two new sets of variables:

$$
\begin{aligned}
& Y_{i t}^{m}=\sum_{k \text { st } \mathcal{A}_{m k}=i} y_{k t}=\left\{\begin{array}{l}
1 \text { if the resource is setup for the value } i \text { of } \\
\text { attribut } m \text { in period } t \\
0 \quad \begin{array}{l}
\text { otherwise. }
\end{array}
\end{array}\right. \\
& Z_{i t}^{m}=\sum_{j \in\left[0, V^{m}\right] \text { st } j \neq i} w_{j i t}^{m}=\left\{\begin{array}{l}
1 \text { if a startup for the value } i \text { of attribut } m \\
\text { takes place at the beginning of period } t \\
0 \begin{array}{l}
\text { otherwise. }
\end{array}
\end{array}\right.
\end{aligned}
$$

With this notation, we have: 
Proposition 1. All feasible solutions of DLSP2 satisfy:

$$
\begin{aligned}
& \forall t, \forall k, \forall p \in\left\{0 \ldots \tilde{D}_{k, t+1, T}\right\}, \forall m=1 \ldots M, \\
& I_{k t} \geq \sum_{q=1}^{p}\left(1-Y_{\mathcal{A}_{m k}, t+q}^{m}-\sum_{\tau=t+q+1}^{S_{k, \tilde{D}_{k, 1, t}+q}} Z_{\mathcal{A}_{m k}, \tau}^{m}\right)
\end{aligned}
$$

Proof. Before the proof, which extends the one given in [30] for the DLSP1 formulation, we briefly explain the idea underlying (19). $Y_{\mathcal{A}_{m k}, t+q}^{m}+\sum_{\tau=t+q+1}^{S_{k, \tilde{D}_{k, 1, t+q}}} Z_{\mathcal{A}_{m k}}=0$ if and only if the resource is not setup in period $t+q$ for the value $\mathcal{A}_{m k}$ of attribute $m$ needed to produce item $k$ and no startup for this value occurs between the period $t+q+1$ and the period where the $q^{\text {th }}$ demand after period $t$ occurs, i.e. if and only if no production of item $k$ is possible in the interval $\left\{t+q, \ldots, S_{k, \tilde{D}_{k, 1, t}+q}\right\}$. In that case, the quantity needed to satisfy the $q^{\text {th }}$ demand on item $k$ after period $t$ should be in stock at the end of period $t$.

Now consider an arbitrary integral feasible solution of DLSP2, say $(y, w, Y, Z)$. We arbitrarily choose an item $k$, a period $t$, a demand occurrence $p \in\left\{0 \ldots \tilde{D}_{k, t+1, T}\right\}$ and an attribute $m$ and we show that the feasible solution chosen satisfies the corresponding valid inequality. In the sequel, for the sake of simplicity, we drop the item index $k$ and we denote $i=\mathcal{A}_{m k}$ the value of attribute $m$ for item $k$.

We denote $R_{q}$ the $q^{\text {th }}$ production period for this item in the feasible solution considered. By definition, we have $R_{1}<R_{2}<\ldots<R_{q}<\ldots<R_{\tilde{D}_{1, T}}$. Moreover, because backlogging is not allowed, the $q^{\text {th }}$ production period must occur before the $q^{\text {th }}$ demand period: $\forall q, R_{q} \leq S_{q}$.

Let $q_{0}$ be the highest index such that $R_{\tilde{D}_{1, t}+q}<t+q$. Then we have:

- $\forall q \leq q_{0}, R_{\tilde{D}_{1, t}+q}<t+q$. The $q^{t h}$ demand after period $t$ is produced before period $t+q$.

- $\forall q>q_{0}, t+q \leq R_{\tilde{D}_{1, t}+q} \leq S_{\tilde{D}_{1, t}+q}$. The $q^{t h}$ demand after period $t$ is produced between $t+q$ and the period $S_{\tilde{D}_{1, t}+q}$ where it occurs. In this case, the resource must be setup for the value $i$ of attribute $m$ at least once in the interval $\left\{t+q, \ldots, S_{\tilde{D}_{1, t}+q}\right\}$. Thus we have:

$$
\forall q>q_{0}, Y_{i, t+q}^{m}+\sum_{\tau=t+q+1}^{S_{\tilde{D}_{1, t}+q}} Z_{i, \tau}^{m} \geq 1
$$


Hence,

$$
\begin{aligned}
\sum_{t=1}^{t} y_{t} & +\sum_{q=1}^{p}\left(Y_{i, t+q}^{m}+\sum_{\tau=t+q+1}^{S_{\tilde{D}_{1, t}+q}} Z_{i, \tau}^{m}\right) \\
& \geq \sum_{t=1}^{t} y_{t}+\sum_{q=1}^{q_{0}} Y_{i, t+q}^{m}+\sum_{q=q_{0}}^{p}\left(Y_{i, t+q}^{m}+\sum_{\tau=t+q+1}^{S_{\tilde{D}_{1, t}+q}} Z_{i, \tau}^{m}\right) \\
& \geq \sum_{t=1}^{t} y_{t}+\sum_{q=1}^{q_{0}} y_{t+q}+p-q_{0} \\
& \geq \tilde{D}_{1, t}+q_{0}+p-q_{0} \\
& \geq \tilde{D}_{1, t}+p
\end{aligned}
$$

(21) comes from the fact that $\sum_{q=1}^{q_{0}} \sum_{\tau=t+q+1}^{S_{\tilde{D}_{1, t}+q}} Z_{i, \tau}^{m} \geq 0$. To obtain (22), we use the fact that $y_{t+q} \leq Y_{i, t+q}^{m}$ as well as the inequalities (20). Finally, (23) is true because, by definition of $q_{0}$, the cumulated demand $\tilde{D}_{1, t}+q_{0}$ is satisfied by the cumulated production before $t+q_{0}, \sum_{t=1}^{t+q_{0}} y_{t}$, so that $\sum_{t=1}^{t+q_{0}} y_{t} \geq \tilde{D}_{1, t}+q_{0}$.

As $\sum_{t=1}^{t} y_{t}-\tilde{D}_{1, t}$ is the inventory level of item $k$ at the end of period $t$, this establishes the validity of (19).

The number of valid inequalities (10) and (19) grows quite fast with the problem size and the production capacity utilization: e.g. for the instances involving 30 products and 100 periods with a capacity utilization of $90 \%$ (see section 5), there are more than 7000 valid inequalities (10) for the DLSP1 formulation and more than 21000 valid inequalities (19) for the DLSP2 formulation. Hence it is not possible to include directly all valid inequalities in the formulations DLSP1 and DLSP2. In the computational experiments to be presented in section 5, the following cutting-plane generation strategy has been implemented to strengthen both formulations:

1. We solve the linear relaxation of the problem using the formulation DLSP1 (respectively DLSP2).

2. We look at each valid inequality of type (10) (respectively (19)). If it is violated by the current continuous solution, we add it to the formulation.

3. If at least one violated inequality is found in step 2, we go back to step 1 and repeat until no more violated valid inequalities can be generated.

The resulting strengthened formulation is denoted DLSP1* (respectively DLSP2*). 


\subsection{A small illustrative example}

We use a small example to illustrate the proposed model and to show an application of the DLSP2 formulation. We consider a bottle filling line where 4 items can be produced. An item is described by the corresponding bottle size (attribute 1 with two possible values) and the composition of the liquid to be used (attribute 2 with two possible values). Table 1 shows how each of the 4 items can be described using the two attributes. We agree to use the item $k=0$ described by the 2 -tuple $(0,0)$ to represent the idle period. Table 2 gives the changeover costs for each attribute and table 3 provides the demand for each product.

Figure 1 shows the optimal production plan obtained while using the DLSP2 formulation. The first two lines give the sequence of setup states for each attribute. In each planning period, we can deduce from these sequences the item for which the resource is setup. The changeover costs to be incurred between each lot are also shown in the bottom line of figure 1. Consistently with our assumption, changeover costs relative to different attributes are added whenever transitions for different attributes occur simultaneously. This is the case here at the beginning of periods 1, 4, 9 and 10 where both the bottle size and the liquid composition are changed.

\section{Computational results}

In this section, we discuss the results of some computational experiments carried out to compare the two formulations presented in sections 2 and 4 . We created 5 sets of randomly generated instances. The instances differ with respect to the following characteristics:

- Problem dimension: The problem dimension is represented by the number of products $N$ and the number of periods $T$. We use three different combinations:

$(N, T) \in\{(10,60),(25,50),(30,100)\}$.

- Multi-attribute product structure: The product structure is described by the number of attributes $M$ and the number of possible values $V^{m}$ for each attribute $m$. We use five different combinations, leading to 5 sets of instances. Table 4 gives the characteristics of the generated instances for each set. For set $\mathrm{C}$ and E instances, we have $\prod_{m=1}^{M} V^{m}>N$. Therefore we used the following procedure to generate matrix $\mathcal{A}$ :

1. We generated a matrix $\mathcal{A}^{\prime}$ with $\prod_{m=1}^{M} V^{m}$ columns. $\mathcal{A}^{\prime}$ describes all possible combinations of the attribute values.

2. For each column $i$ of $\mathcal{A}^{\prime}$, we randomly generated a weight $w_{i}$ from a discrete 
uniform $D U\left(1, \prod_{m=1}^{M} V^{m}\right)$ distribution.

3. The matrix $\mathcal{A}$ is generated by selecting the $N$ columns of $\mathcal{A}^{\prime}$ corresponding to the $N$ smallest weight $w_{i}$.

- Inventory holding costs: For each item, inventory holding costs have been generated randomly from a discrete uniform $D U(5,10)$ distribution.

- Production capacity utilization: Production capacity utilization $\rho$ is defined as the ratio of the total cumulated demand on the total cumulated available capacity. We experimented different values for $\rho$ : $0.5,0.7$ and 0.9 .

- Demand pattern: Binary demands for each item have been randomly generated according to the procedure described in [24].

- Changeover costs: For each attribute $m$, changeover costs $C_{l k}^{m}$ have been randomly generated from a discrete uniform $D U\left(C_{m i n}^{m}, C_{m a x}^{m}\right)$ distribution. We tested several possibilities: the changeover costs for all attributes can either be taken from the same interval or the changeover costs for the first attribute are greater than for the other(s). In our study, we define the ratio $r$ as: $r=\frac{C_{\text {mean }}^{1}}{C_{\text {mean }}^{m}}$ where $C_{\text {mean }}^{m}$ denotes the mean of interval $\left[C_{m i n}^{m}, C_{m a x}^{m}\right]$. We tested several values for $r: 1,2,5,10$ and 30. In all instances, the resulting changeover costs between two items belong to the interval $[0,200]$.

For each possible combination of multi-attribute product structure, production capacity utilization and changeover costs ratio, 5 problems were generated, resulting in $5 \times 3 \times 5 \times$ $5=375$ instances. All tests were run on a Pentium 4 (2.8 Ghz) with $505 \mathrm{Mb}$ of RAM, running under Windows XP. We used a standard MIP software (CPLEX 8.1.0) with the solver default settings, using either formulation DLSP1 or formulation DLSP2.

Tables 5-9 show the computational results obtained with formulations DLSP1* and DLSP2 $^{*}$, for each set of instances. As the value of the ratio $r$ appears to have an impact on the results quality, we grouped the instances with respect to the value of $r$ so that each line corresponds to the average value for 15 randomly generated instances ( 5 instances for each value of production capacity utilization). For both series of results, we provide:

- \#Opt: for set $\mathrm{A}, \mathrm{B}$ and $\mathrm{C}$ instances, the number of instances out of the corresponding 15 instances that could be solved to optimality within 30 minutes of computation.

- \#Feas: for set D and E instances, the number of instances out of the corresponding 15 instances for which a feasible solution could be found within 30 minutes of computation. 
- Gap: for the instances that could not be solved to optimality, the average relative gap value obtained after 30 minutes of computation between the best integer solution (if one could be found) and the best lower bound found.

We now compare the results obtained with formulations DLSP1* and DLSP2*. The results from tables 5-9 show that:

- for high values of ratio $r(r \geq 5)$, i.e. when one attribute has corresponding changeover costs clearly higher than the other(s) attribute(s), the results obtained with formulation DLSP2* are better. This can be seen as:

- a feasible solution could be obtained for all instances,

- more instances could be solved to optimality within 30 minutes of computation,

- when a guaranteed optimal solution could not be found within 30 minutes of computation, the residual gap is smaller.

- for small values of the ratio $r(r \leq 2)$, formulation DLSP1* provides better results for medium-sized instances (sets A, B and C). However, this is not the case for the larger instances in sets D and E. Namely, for these instances,

- a feasible solution could not always be found with formulation DLSP1* whereas at least one feasible solution could be found for each instance with formulation DLSP2*.

- the residual gap is significantly smaller on some instances with formulation DLSP2*.

Comparison between the results obtained with the two formulations thus shows that using formulation DLSP2*, we are able to improve the efficiency of the Branch \& Bound procedure, especially for the high values of ratio $r$ and for the largest instances. This can be explained by two main factors:

- Using formulation DLSP2*, the problem size (i.e. the number of variables and constraints) is significantly reduced. As a consequence, the time spent at each node of the Branch \& Bound tree to solve the linear relaxation is shorter and more nodes can be explored within 30 minutes of computation.

- The formulation enhancement obtained thanks to the valid inequalities adapted for formulation DLSP2 gives better results when ratio $r$ has a high value. More precisely, for high values of $r$, the lower bounds provided by formulation DLSP2* are higher than the ones provided by formulation DLSP1*. On the contrary, for 
small values of $r$, the lower bounds provided by the formulation DLSP1* are higher than the ones provided by formulation DLSP2*.

Thus the combined advantages of a reduced problem size and of tighter lower bounds enable the formulation DLSP2* to outperform the formulation DLSP1* on many instances.

\section{Conclusion}

We presented a new formulation for the DLSP with sequence-dependent setup costs. The main idea is to use a possible product description as combinations of a number of physical attributes. When such a structure is present in the industrial context under study, we have shown how to exploit it to reduce the size of the mixed integer linear program to be solved and improve the efficiency of the solution process. Computational experiments show that the proposed DLSP2 formulation performs better than the tight DLSP1 formulation we chose as a reference for comparison, especially in cases where one of the physical attributes has corresponding changeover costs higher than the other(s) attribute(s).

Finally, future research on this topic could be aimed at extending the proposed MIP formulation DLSP2* to take into consideration additional relevant industrial concerns such as the presence of multiple resources that need to be planned simultaneously (see e.g. [3], [18], [22] or [25]) or the integration of positive changeover times (see e.g. [14], [24], [28]or [29]). It would also be worth investigating whether a multi attribute product structure could be used to solve lot sizing problems such as the ELSP where different modelling assumptions are used. 


\section{References}

[1] Belvaux G and Wolsey L. Modelling practical lot-sizing problems as mixed-integer programs. Management Science 2001; 47(7): 993-1007

[2] Bomberger E. A dynamic programming approach to a lot size scheduling problem. Management Science 1966; 12(11): 778-784

[3] Dastidar SG and Nagi R. Scheduling injection molding operations with multiple resource constraints and sequence dependent setup times and costs. Computers \& Operations Research 2005; 32: 2987-3005

[4] Dobson G. The cyclic lot scheduling problem with sequence-dependent setups. Operations Research 1992; 40(4): 736-749

[5] dos Santos-Meza E, dos Santos M and Arenales MN. A lot-sizing problem in an automated foundry. European Journal of Operational Research 2002; 139: 490-500

[6] Drexl A and Kimms A. Lot sizing and scheduling - Survey and extensions. European Journal of Operational Research 1997; 99: 221-235

[7] Elmaghraby S. The Economic Lot Scheduling problem (ELSP): review and extensions. Management Science 1978; 24(6): 587-598

[8] Fleischmann B. The discrete lot sizing and scheduling problem. European Journal of Operational Research 1990; 44: 337-348

[9] Fleischmann B. The discrete lot sizing and scheduling problem with sequencedependent set-up costs. European Journal of Operational Research 1994; 75: 395-404

[10] Gao L, Altay N and Robinson EP. A comparative study of modeling and solution approaches for the coordinated lot-size problem with dynamic demand. Mathematical and Computer Modelling 2008; 47: 1254-1263

[11] Ho JC and Chang Y. A new MRP/GT lot sizing heuristic: a simulation study. Production Planning \& Control 2003; 14(3): 215-224

[12] Jans R and Degraeve Z. Meta-heuristics for dynamic lot sizing: a review and comparison of solution approaches. European Journal of Operational Research 2007; 177: $1855-1875$

[13] Jans R and Degraeve Z. Modelling industrial lot sizing problems: a review. International Journal of Production Research 2008; 46(6): 1619-1643 
[14] Jordan C and Drexl A. Discrete Lotsizing and Scheduling by Batch Sequencing. Management Science 1998; 44(5): 698-713

[15] Karmarkar U and Schrage L. The deterministic dynamic product cycling problem. Operations Research 1985; 33(2): 326-345

[16] Kuik R, Salomon M and van Wassenhove LN. Batching decisions: structure and models. European Journal of Operational Research 1994; 75: 243-263

[17] Meyr H. Simultaneous lotsizing and scheduling by combining local search with dual reoptimization. European Journal of Operational Research 2000; 120: 311-326

[18] Meyr H. Simultaneous lotsizing and scheduling on parallel machines. European Journal of Operational Research 2002; 139: 277-292

[19] Miègeville N. Supply Chain Optimization in the process industry. Methods and Case Study of the Glass Industry. PhD dissertation 2005; Ecole Centrale Paris, France

[20] Mohamed ZM, Youssef MA and Huq F. An efficient model for multifamily lot-sizing and scheduling: application to a real life problem. Production Planning \& Control 2004; 15(1): 90-101

[21] Oh $\mathrm{H}$ and Karimi IA. Planning production on a single processor with sequencedependent setup part 1: determination of campaigns. Computers \& Chemical Engineering 2001; 25: 1021-1030

[22] Özdamar L and Birbil SI. Hybrid heuristics for the capacitated lot sizing and loading problem with setup times and overtime decisions. European Journal of Operational Research 1998; 110: 525-547

[23] Robinson EP and Lawrence F.B. Coordinated capacitated lot-sizing problem with dynamic demand: a lagrangian heuristic. Decision Sciences 2004; 35(1): 25-53

[24] Salomon M, Solomon M, van Wassenhove L, Dumas Y and Dauzère-Pérès S. Solving the discrete lotsizing and scheduling problem with sequence dependent set-up costs and set-up times using the Travelling Salesman Problem with time windows. European Journal of Operational Research 1997; 100: 494-513

[25] Segerstedt A. A capacity-constrained multi-level inventory and production control problem. International Journal of Production Economics 1996; 45: 449-461

[26] Silva C and Magalhaes JM. Heuristic lot size scheduling on unrelated parallel machines with applications in the textile industry. Computers \& Industrial Engineering 2006; 50: 76-89 
[27] Smith-Daniels V.L. and Smith-Daniels DE. A mixed integer programming model for lot sizing and sequencing packaging lines in the process industries. IIE Transactions 1986; 18: 278-285

[28] Trigeiro W, Thomas L and McClain J. Capacitated lot sizing with setup times. Management Science 1989; 35(3): 353-366

[29] Vanderbeck F. Lot-sizing with start-up times. Management Science 1998; 44(10): $1409-1425$

[30] van Eijl CA and van Hoesel CPM. On the discrete lot-sizing and scheduling problem with Wagner-Whitin costs. Operations Research Letters 1997; 20: 7-13

[31] Wagner BJ and Davis DJ. A search heuristic for the sequence-dependent economic lot scheduling problem. European Journal of Operational Research 2002; 141(1): 133146

[32] Wolsey L. Solving multi-item lot-sizing problems with an MIP solver using classification and reformulation. Management Science 2002; 48(12): 1587-1602

[33] Zhu XY and Wilhelm WE. Scheduling and lot sizing with sequence-dependent setup: A literature review. IIE Transactions 2006; 38(11): 987-1007 


\begin{tabular}{|c|c|c|c|c|c|c|c|c|c|c|c|}
\hline Period & 0 & 1 & 2 & 3 & 4 & 5 & 6 & 7 & 8 & 9 & 10 \\
\hline Bottle size & 0 & 1 & 1 & 1 & 2 & 2 & 2 & 2 & 2 & 0 & 1 \\
\hline $\begin{array}{l}\text { Liquid } \\
\text { composition }\end{array}$ & 0 & 1 & 1 & 1 & 2 & 1 & 1 & 1 & 1 & 0 & 2 \\
\hline Item & $(0,0)$ & $(1,1)$ & $(1,1)$ & $(1,1)$ & $(2,2)$ & $(2,1)$ & $(2,1)$ & $(2,1)$ & $(2,1)$ & $(0,0)$ & $(1,2)$ \\
\hline Lot schedule & & & Item 1 & & Item 4 & & & & & Idle & Item 2 \\
\hline $\begin{array}{l}\text { Changeover } \\
\text { costs }\end{array}$ & & 10 & & & 20 & & & & & & 10 \\
\hline
\end{tabular}

Figure 1: Optimal production plan for the simple example: optimal cost $Z^{*}=528$ 


\begin{tabular}{l|ccccc} 
item & 0 & 1 & 2 & 3 & 4 \\
\hline attribute 1: bottle size & 0 & 1 & 1 & 2 & 2 \\
attribute 2: liquid composition & 0 & 1 & 2 & 1 & 2 \\
\hline product description & $(0,0)$ & $(1,1)$ & $(1,2)$ & $(2,1)$ & $(2,2)$ \\
\hline inventory holding costs & 0 & 7 & 8 & 5 & 10
\end{tabular}

Table 1: Product description using two attributes

\begin{tabular}{c|ccc} 
& 0 & 1 & 2 \\
\hline 0 & 0 & 100 & 200 \\
1 & 0 & 0 & 200 \\
2 & 0 & 100 & 0
\end{tabular}

\begin{tabular}{c|ccc} 
& 0 & 1 & 2 \\
\hline 0 & 0 & 10 & 10 \\
1 & 0 & 0 & 20 \\
2 & 0 & 10 & 0
\end{tabular}

Table 2: Changeover costs for transitions between bottle sizes (left) and between liquid composition (right)

\begin{tabular}{c|cccccccccc} 
period & 1 & 2 & 3 & 4 & 5 & 6 & 7 & 8 & 9 & 10 \\
\hline item 1 & 0 & 1 & 0 & 0 & 1 & 0 & 0 & 1 & 0 & 0 \\
item 2 & 0 & 0 & 0 & 0 & 0 & 0 & 0 & 0 & 0 & 1 \\
item 3 & 0 & 0 & 0 & 0 & 1 & 1 & 0 & 1 & 0 & 1 \\
item 4 & 0 & 0 & 0 & 1 & 0 & 0 & 0 & 0 & 0 & 0
\end{tabular}

Table 3: Demand on products

\begin{tabular}{l|cccl} 
& $\mathrm{N}$ & $\mathrm{T}$ & $\mathrm{M}$ & $V^{m}$ \\
\hline set A & 10 & 60 & 2 & $V^{1}=2, V^{2}=5$ \\
set B & 25 & 50 & 2 & $V^{1}=5, V^{2}=5$ \\
set C & 25 & 50 & 3 & $V^{1}=3, V^{2}=3, V^{3}=3$ \\
set D & 30 & 100 & 3 & $V^{1}=2, V^{2}=3, V^{3}=5$ \\
set $\mathrm{E}$ & 30 & 100 & 5 & $V^{1}=2, V^{2}=2, V^{3}=2, V^{4}=2, V^{5}=2$
\end{tabular}

Table 4: Multi-attribute product structure: characteristics of generated instances 
Formulation DLSP1* Formulation DLSP2*

\begin{tabular}{ccccc}
\hline $\begin{array}{c}\text { Variables } \\
\text { Constraints }\end{array}$ & \multicolumn{2}{c}{8520} & \multicolumn{2}{c}{3960} \\
\#VI & \multicolumn{1}{l}{1921} & \multicolumn{2}{c}{1681} \\
ratio $r$ & \#Opt & Gap & \#Opt & Gap \\
\hline$r=1$ & 13 & $3 \%$ & 5 & $9 \%$ \\
$r=2$ & 10 & $6 \%$ & 5 & $5 \%$ \\
$r=5$ & 2 & $12 \%$ & 6 & $4 \%$ \\
$r=10$ & 0 & $21 \%$ & 11 & $3 \%$ \\
$r=30$ & 1 & $14 \%$ & 15 & $0 \%$ \\
\hline
\end{tabular}

Table 5: Results for set A instances

Formulation DLSP1* Formulation DLSP2*

\begin{tabular}{ccccc}
\hline $\begin{array}{c}\text { Variables } \\
\text { Constraints } \\
\text { \#VI }\end{array}$ & \multicolumn{2}{c}{36350} & \multicolumn{2}{l}{6150} \\
& 8381 & \multicolumn{2}{c}{2451} \\
ratio $r$ & \#Opt & Gap & \#Opt & Gap \\
\hline$r=1$ & 3 & $7 \%$ & 0 & $17 \%$ \\
$r=2$ & 0 & $16 \%$ & 0 & $16 \%$ \\
$r=5$ & 0 & $26 \%$ & 4 & $11 \%$ \\
$r=10$ & 0 & $31 \%$ & 7 & $4 \%$ \\
$r=30$ & 0 & $36 \%$ & 11 & $5 \%$ \\
\hline
\end{tabular}

Table 6: Results for set B instances

Formulation DLSP1* Formulation DLSP2*

\begin{tabular}{ccccc}
\hline $\begin{array}{c}\text { Variables } \\
\text { Constraints }\end{array}$ & \multicolumn{2}{c}{36350} & \multicolumn{2}{c}{4950} \\
\#VI & \multicolumn{2}{c}{3851} & \multicolumn{2}{c}{2451} \\
& 840 & \multicolumn{2}{c}{1653} \\
\hline ratio $r$ & \#Opt & Gap & \#Opt & Gap \\
\hline$r=1$ & 5 & $11 \%$ & 0 & $20 \%$ \\
$r=2$ & 3 & $13 \%$ & 0 & $21 \%$ \\
$r=5$ & 0 & $24 \%$ & 0 & $14 \%$ \\
$r=10$ & 0 & $24 \%$ & 9 & $10 \%$ \\
$r=30$ & 0 & $39 \%$ & 8 & $7 \%$ \\
\hline
\end{tabular}

Table 7: Results for set $\mathrm{C}$ instances 
Formulation DLSP1* Formulation DLSP2*

\begin{tabular}{ccccc}
\hline Variables & \multicolumn{2}{c}{102200} & \multicolumn{2}{c}{12200} \\
Constraints & 9201 & \multicolumn{2}{c}{5601} \\
\#VI & \multicolumn{2}{c}{2792} & \multicolumn{2}{c}{2865} \\
\hline ratio $r$ & \#Feas & Gap & \#Feas & Gap \\
\hline$r=1$ & 10 & $38 \%$ & 15 & $40 \%$ \\
$r=2$ & 7 & $42 \%$ & 15 & $42 \%$ \\
$r=5$ & 10 & $48 \%$ & 15 & $35 \%$ \\
$r=10$ & 10 & $57 \%$ & 15 & $29 \%$ \\
$r=30$ & 10 & $62 \%$ & 15 & $24 \%$ \\
\hline
\end{tabular}

Table 8: Results for set D instances

Formulation DLSP1* Formulation DLSP2*

\begin{tabular}{ccccc}
\hline Variables & \multicolumn{2}{c}{102200} & \multicolumn{2}{c}{$\begin{array}{l}10600 \\
\text { Constraints }\end{array}$} \\
\#VI & \multicolumn{2}{c}{2792} & \multicolumn{2}{c}{$\begin{array}{l}6001 \\
2911\end{array}$} \\
\hline ratio $r$ & \#Feas & Gap & \#Feas & Gap \\
\hline$r=1$ & 12 & $29 \%$ & 15 & $31 \%$ \\
$r=2$ & 10 & $34 \%$ & 15 & $36 \%$ \\
$r=5$ & 9 & $47 \%$ & 15 & $33 \%$ \\
$r=10$ & 10 & $54 \%$ & 15 & $29 \%$ \\
$r=30$ & 12 & $59 \%$ & 15 & $26 \%$ \\
\hline
\end{tabular}

Table 9: Results for set E instances 